\title{
ANTONOV WETTING LINE PHASE TRANSITION OF TWO-COMPONENT BOSE-EINSTEIN CONDENSATES UNDER CONSTRAINT OF ROBIN BOUNDARY CONDITION
}

\author{
Nguyen Van Thu ${ }^{a^{*}}$, Hoang Van Quyet ${ }^{\mathrm{a}}$ \\ ${ }^{a}$ The Faculty of Physics, Hanoi Pedagogical University 2, Hanoi, Vietnam \\ *Corresponding author: Email: nvthu@live.com
}

Article history

Received: January $14^{\text {th }}, 2018$

Received in revised form: April 09 ${ }^{\text {th }}, 2018 \mid$ Accepted: April 25 ${ }^{\text {th }}, 2018$

\begin{abstract}
Using double Parabola approximation, in this paper, after finding the wave function for the ground state, we found an analytical relation for wetting phase transition and Antonov line of two-component Bose-Einstein condensates. The Robin boundary condition was applied for our system. Based on these results, we reobtained results for our system with constraint by Dirichlet boundary condition.
\end{abstract}

Keywords: Antonov line; Bose-Einstein condensates; Double parabola approximation; Ground state; Robin boundary condition; Wetting phase transition.

Article identifier: http://tckh.dlu.edu.vn/index.php/tckhdhdl/article/view/401

Article type: (peer-reviewed) Full-length research article

Copyright $@ 2018$ The author(s).

Licensing: This article is licensed under a CC BY-NC-ND 4.0 


\title{
ĐƯÒ̀NG CHUYỂN PHA DÍNH ƯỚT ANTONOV CỦA NGƯNG TỤ BOSE-EINSTEIN HAI THÀNH PHÀ̀N VỚI ĐIỀU KIỆN BIÊN ROBIN
}

\author{
Nguyễn Văn Thụ ${ }^{a^{*}}$, Hoàng Văn Quyết ${ }^{\mathrm{a}}$
}

${ }^{a}$ Khoa Vật lý, Truờng Đại học Su phạm Hà Nội 2, Hà Nội, Việt Nam

"Tác giả liên hệ: Email: nvthu@live.com

Lịch sử bài báo

Nhận ngày 14 tháng 01 năm 2018

Chỉnh sửa ngày 09 tháng 04 năm 2018 | Chấp nhận đăng ngày 25 tháng 04 năm 2018

\section{Tóm tắt}

Sư dụng gần đúng Parabol kép, trong bài báo này, sau khi tìm được hàm sóng cho trạng thái co bản, chúng tôi tìm được biểu thức giải tích cho đường chuyển pha ướt Antonov của hệ ngung tu Bose-Einstein hai thành phần. Điều kiện biên được sử dụng cho hệ là điều kiện biên Robin, trên cơ sở đó chúng tôi cung thu lại được kết quả tuơng ứng cho hệ với điều kiện biên Dirichlet.

Từ khóa: Chuyển pha ướt; Điều kiện biên Robin; Đường chuyển pha Antonov; Gần đúng parabol kép; Ngưng tụ Bose-Einstein; Trạng thái cơ bản.

Mã số định danh bài báo: http://tckh.dlu.edu.vn/index.php/tckhdhdl/article/view/401

Loại bài báo: Bài báo nghiên cứu gốc có bình duyệt

Bản quyền @ 2018 (Các) Tác giả.

Cấp phép: Bài báo này được cấp phép theo CC BY-NC-ND 4.0 


\section{INTRODUCTION}

Wetting phase transition is the most important phenomenon in the field of study on Bose-Einstein condensates (BECs), especially its applications in technology. This fact was investigated for the first time by Indekeu and Schaeybroeck (2004). Then it has opened up a new avenue for physicists in this scope. Based on this work, the wetting phenomena have been widely studied. In Indekeu and Schaeybroeck (2015), wetting phase transition is considered for an optical wall and second component wets this wall. The authors also predicted relation for wetting line. After double parabola approximation (DPA) was proposed by Indekeu, Lin, Nguyen, Schaeybroeck, and Tran (2015). Nguyen (2016) proved thoroughly the relation for Antonov line. However, these studies only concentrate on Dirichlet boundary condition (BC). However, in electronic technology, several BCs are required in some given cases. For example, Robin BC is applied when one uses capillary wave at the interface.

The main aim of this paper is considering effects from Robin BC to Antonov line phase transition of BECs in semi-infinite space. To do this, we started from the GP Hamiltonian in the bulk of a BECs without the external trapping potential (Pethick \& Smith, 2008).

$$
H=\sum_{j=1,2} \psi_{j}^{*}\left(-\frac{\mathrm{h}^{2}}{2 m_{j}} \nabla^{2}\right) \psi_{j}+V\left(\psi_{1}, \psi_{2}\right),
$$

in which Gross-Pitaevskii (GP) potential

$$
V\left(\psi_{1}, \psi_{2}\right)=\sum_{j=1,2}\left[-\mu_{j}|\psi|^{2}+\frac{g_{j j}}{2}\left|\psi_{j}\right|^{4}\right]+g_{12}\left|\psi_{1}\right|^{2}\left|\psi_{2}\right|^{2},
$$

where $\psi_{j}, m_{j}$, and $\mu_{j}$ are the wave function, the atomic mass and the chemical potential of each species $j$, respectively. The interaction constants are defined via $s$-wave scattering length $a_{i j j^{\prime}}$ between components $j$ and $j^{\prime}$ by $g_{j j^{\prime}}=2 \pi \mathrm{h}^{2}\left(1 / m_{j}+1 / m_{j^{\prime}}\right) a_{i j j^{\prime}}>0$. In order to make sure that the wetting phenomena occur, we only consider the case two components are immiscible, i. e. $g_{12}^{2}>g_{11} g_{22}$.

\section{THE ANTONOV WETTING LINE PHASE TRANSITION}

\subsection{Ground state}

We first find the wave function for the ground state. The system under consideration is translational symmetry in the $x-y$ direction and restricted by a wall at $z=0$. To sake the simplicity, one introduces the dimensionless coordinate $\rho=z / \xi_{1}$ with $\xi_{j}=\mathrm{h} / \sqrt{2 m_{j} g_{j j} n_{0 j}}$ being healing length and $n_{0 j}$ is bulk density of component $j$. The 
chemical potential, in grand canonical ensemble, has the form $\mu_{j}=g_{j j} n_{0 j}$. The Hamiltonian (1) and GP potential (2) are reduced to

$$
\begin{aligned}
& \mathbb{H}=\frac{H}{2 P_{0}}=-\sum \phi_{j} \partial_{\rho}^{2} \phi_{j}+V_{G P}, \\
& V_{G P}=\sum_{j=1,2}\left(-\phi_{j}^{2}+\frac{\phi_{j}^{4}}{2}\right)+K \phi_{1}^{2} \phi_{2}^{2},
\end{aligned}
$$

where $K=g_{12} / \sqrt{g_{11} g_{22}}, \phi_{j}=\psi_{j} / \sqrt{n_{0 j}}$ and $P_{0}=g_{j j} n_{0 j}^{2} / 2$ is pressure, which takes one and the same value in both condensates at two-phase coexistence. The equilibrium values of the order parameters $\phi_{j}$ minimize the total Hamiltonian given in equation (3) and (4). That allows us to derive immediately the time-independent GP equations

$$
-\partial_{\rho}^{2} \phi_{j}-\phi_{j}+\phi_{j}^{3}+K \phi_{j} \phi_{j^{\prime}}^{2}=0
$$

In order to get the analytical solution for these equations, we employ the DPA. One assumes that component 1 (2) occupies the region $\rho>1(\rho<1)$. Here $L$ denotes position of the interface. Expanding the order parameters about bulk condensate $1,\left(\phi_{1}, \phi_{2}\right)=(1,0)$ for half-space $\rho>1$ and bulk condensate $2\left(\phi_{1}, \phi_{2}\right)=(0,1)$ for the remaining half-space, the GP potential (4) becomes DPA potential

$$
V_{D P A}=\alpha^{2}\left(\phi_{j}-1\right)^{2}+\beta^{2} \phi_{j^{\prime}}^{2}-1 / 2
$$

where $\alpha=\sqrt{2}, \beta=\sqrt{K-1}$. The labels $j$ and $j$ ' comply with the following important convention, which we will henceforth maintain throughout this paper: $\left(j, j^{\prime}\right)=(1,2)$ if $\rho>1$ and vice versa. Within DPA, equation (5) reduces to

$$
\begin{aligned}
& -\partial_{\rho}^{2} \phi_{j}+\alpha^{2}\left(\phi_{j}-1\right)=0 \\
& -\xi^{2} \partial_{\rho}^{2} \phi_{j^{\prime}}+\beta^{2} \phi_{j^{\prime}}=0 .
\end{aligned}
$$

Here we denote $\xi=\xi_{2} / \xi_{1}$. In our previous work (Nguyen, Tran, \& Pham, 2016), we proved that the boundary condition (BC) is either Dirichlet or Robin. In this paper, the first component is requested by Dirichlet $\mathrm{BC}$ and Robin $\mathrm{BC}$ for the second one

$$
\phi_{1}(0)=0, \phi_{1}(\infty)=1 ; \phi_{2}(0)=\left.\lambda \frac{\partial \phi_{2}}{\partial \rho}\right|_{\rho=0}, \phi_{2}(\infty)=0,
$$


in which $\lambda$ is dimensionless constant. Solving (7), (8) and keeping in mind the continuity of the wave functions and its first derivative at the interface one obtains

$$
\phi_{1}=1-A_{1} e^{-\alpha \rho}, \phi_{2}=B_{1} e^{-\beta \rho / \xi}
$$

for $\rho>1$ and

$$
\phi_{1}=2 A_{2} \sinh (\beta \rho), \phi_{2}=1+B_{2} e^{\alpha \rho / \xi}+\frac{\left[\alpha \lambda B_{2}-\left(B_{2}+1\right) \xi\right]}{\alpha \lambda+\xi}
$$

where

$$
\begin{aligned}
& A_{1}=\frac{\beta e^{\alpha \mathrm{l}}}{\beta+\alpha \tanh (\beta 1)}, B_{1}=\frac{\alpha e^{\beta 1 / \xi}\left(e^{\alpha 1 / \xi}-1\right)\left[\alpha \lambda-\xi+(\alpha \lambda+\xi) e^{\alpha 1 / \xi}\right]}{(\alpha+\beta)(\alpha \lambda+\xi) e^{2 \alpha 1 / \xi}-(\alpha-\beta)(\alpha \lambda-\xi)} \\
& A_{2}=\frac{\alpha \operatorname{csch}(\beta 1)}{2[\alpha+\beta \operatorname{coth}(\beta 1)]}, B_{2}=\frac{\xi(\beta-\alpha)-\beta(\alpha \lambda+\xi) e^{\alpha 1 / \xi}}{(\alpha+\beta)(\alpha \lambda+\xi) e^{2 \alpha 1 / \xi}-(\alpha-\beta)(\alpha \lambda-\xi)}
\end{aligned}
$$

\subsection{Wetting phase transition and Antonov line}

The fundamental of physics for the wetting is Young's law (de Genns, 1985), in which the familiar energy is in balance

$$
\gamma_{W 1}=\gamma_{W 2}+\gamma_{12} \cos \theta
$$

where $\gamma_{W j}$ is the surface energy of a phase of pure component $\mathrm{j}, \gamma_{12}$ is the interfacial tension at the interface and $\theta$ is contact angle. At phase of complete wetting $\theta=0$ thus equation (10) reduced to

$$
\gamma_{W 1}=\gamma_{W 2}+\gamma_{12}
$$

We now calculate the interfacial tension. The grand potential of our system can be written in dimensionless form as follows:

$$
\Omega=2 P_{0} \xi_{1} A \int_{0}^{\infty} d \rho\left(-\phi_{1} \partial_{\rho}^{2} \phi_{1}-\xi^{2} \phi_{2} \partial_{\rho}^{2} \phi_{2}+V\right),
$$

with A being the interface area. Replacing $V$ in (12) by DPA potential (6) and combining with the constant of motion, one leads to the excess grand potential per unit area, which is called interfacial tension 


$$
\gamma_{12}=2 P_{0} \xi_{1} \int_{0}^{\infty} d \rho\left[2\left(\partial_{\rho} \phi_{1}\right)^{2}+(2-\lambda) \xi^{2}\left(\partial_{\rho} \phi_{2}\right)^{2}\right]
$$

Plugging equation (9) into (13) one easily derives an analytical relation for interfacial tension; it is quite large and without insight. In complete wetting phase, it does not depend on 1 and has the form

$$
\gamma_{12}=\frac{\alpha \beta}{\alpha+\beta}+\frac{(2-\lambda) \alpha \xi\left[\alpha^{2} \beta \lambda^{2}+2 \alpha \beta \lambda \xi+(\alpha+2 \beta) \xi^{2}\right.}{(\alpha+\beta)(\alpha \lambda+\xi)^{2}} P_{0} \xi_{1}
$$

Now we define the surface tension (or wall tension) of pure phase $\mathrm{j}$ as the excess energy per unit area (Indekeu \& Schaeybroeck, 2015),

$$
\gamma_{W j, p u r e}=\lim _{L \rightarrow \infty}\left[-P_{1} \xi_{1} \int_{0}^{L} \phi_{j}^{4} d z+P_{j} \xi_{1} \int_{0}^{L} d z\right] .
$$

Assuming $\xi<1$ one can check

$$
\gamma_{W 2, p u r e}=\frac{(2-\lambda) \alpha \xi^{2}}{(\alpha \lambda+\xi)^{2}} P_{0} \xi_{2}
$$

For the first component, as mentioned in Indekeu and Schaeybroeck (2015), we can define wall tension, which is obtained by subtracting from the total grand potential $\Omega$ the grand potential of a half space $\rho>0$ filled with pure phase 1 , both divided by $A$,

$$
\gamma_{W 1}=\lim _{L-\infty}\left[-2 P_{1} \xi_{1} \int_{0}^{\infty} d z\left(\frac{\phi_{1}^{4}}{2}+\frac{\phi_{2}^{4}}{2}+K \phi_{1}^{2} \phi_{2}^{2}\right)+P_{1} \xi_{1} \int_{0}^{L} d z\right]
$$

Combining (15) and (17) we get

$$
\gamma_{W 1}=\gamma_{W 1, \text { pure }}+\gamma_{W 2, \text { pure }}-2 P_{1} \xi_{1} \int_{0}^{\infty} K \phi_{1}^{2} \phi_{2}^{2}
$$

At complete wetting phase, the last term on the right-hand side of equation (18) tends to zero so in coexistence phase one gets

$$
\gamma_{W 1}=\frac{\alpha(2-\lambda)}{(\alpha \lambda+1)^{2}} P_{0}\left(\xi_{1}+\xi_{2}\right)
$$

Inserting (14), (16) and (19) into (11) one has an equation for Antonov line 


$$
\sqrt{K-1}=\frac{1}{\alpha}\left[\frac{1+\alpha \lambda}{\xi}-(1+\alpha \lambda)\right]
$$

It is easy to see that if we set $\lambda=0$, equation (20) will be reduced to Antonov line corresponding to Dirichlet BC in Nguyen (2016).

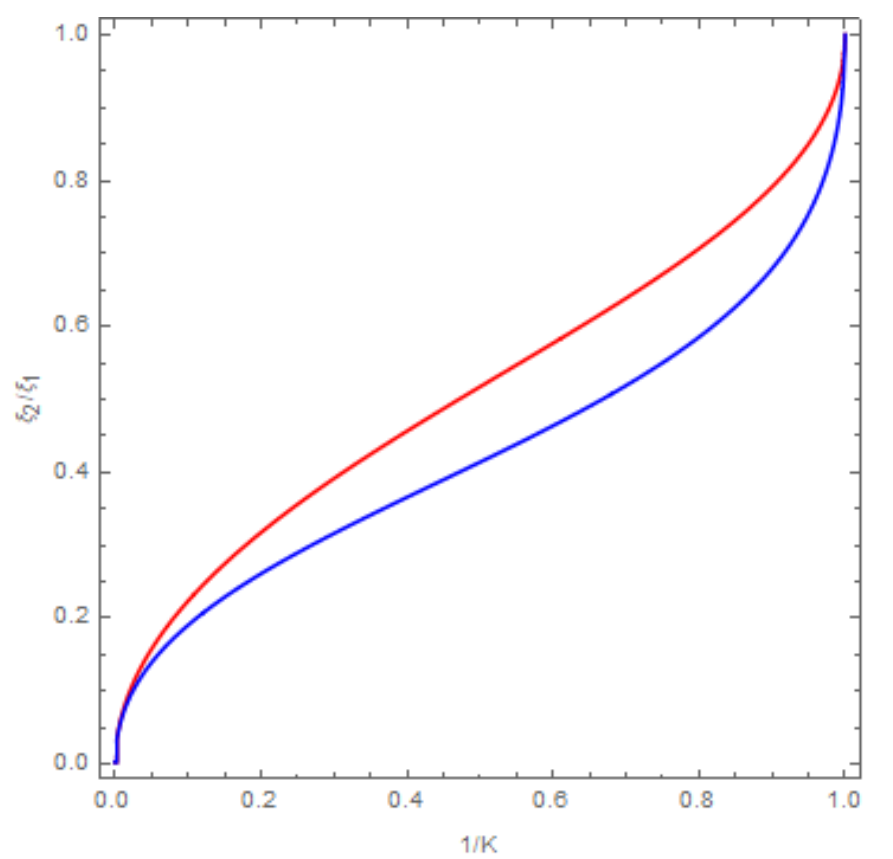

Figure 1. Antonov lines for Robin BC with $\lambda=\xi / \alpha$ (red line) and Dirichlet BC (blue line)

Figure 1 shows the Antonov lines, in which the red and blue lines correspond to Robin BC and Dirichlet BC, respectively. In this figure, we set $\lambda=\xi / \alpha$ associating with Robin BC. It is obvious that there is a significant effect from BC on the Antonov line, especially in middle separation.

\section{CONCLUSION}

In the foregoing section, we presented the main results of our work. In scope of DPA we study the two-component BEC in semi-infinity system with a wall. Our results are in order:

- We found analytical solutions for the ground state with Robin boundary conditions in all kinds of segregation;

- The interfacial tension was considered. In addition, the wall tension for each component was also investigated; 
- The relation for Antonov line of wetting phase transition was obtained.

The constant $\lambda$ corresponding to Robin BC is an interesting quantity, which plays the role of extra-interpolation length and its value depends on the specific system.

\section{REFERENCES}

de Genns, P. G. (1985). Wetting: Statics and dynamics. Review of Modern Physics, 57(3), 827-863.

Indekeu, J. O., Lin, C. Y., Nguyen, V. T., Schaeybroeck, B. V., \& Tran, H. P. (2015). Statistical interfacial properties of Bose-Einstein condensate mixtures. Physical Review A, 91(033615), 1-24.

Indekeu, J. O., \& Schaeybroeck, B. V. (2004). Extraordinary phase diagram for mixtures of Bose-Einstein condensates. Physical Review Letters, 93(21), 1-4.

Indekeu, J. O., \& Schaeybroeck, B. V. (2015). Critical wetting, first-order wetting, and prewetting phase transition in binary mixtures of Bose-Einstein condensates. Physical Review A, 91(013626), 1-18.

Nguyen, V. T. (2016). Static properties of Bose-Einstein condensate mixtures in semiinfinite space. Physics Letters A, 380(37), 2920-2924.

Nguyen, V. T., Tran, H. P., \& Pham, T. S. (2016). Wetting phase transition of two segregated Bose-Einstein condensates restricted by a hard wall. Physics Letters A, 380(16), 1487-1492.

Pethick, C. J., \& Smith, H. (2008). Bose-Einstein condensation in dilute gases. Cambridge, UK: Cambridge University Press. 